\title{
Polymyalgia and low back pain: a common cause not to be missed
}

\author{
N Hopkinson, A A Myint, S Benjamin
}

\section{Case reports}

PATIENT 1

A 65 year old man was admitted with a one month history of increasingly severe left sided sciatica. He had one previous episode of low back pain 40 years earlier. Four months before admission, a left inguinal hernia was repaired and following this he had complained of pain in the left testicle. His pain had rapidly increased day and night despite chiropractic treatment, and he complained of anorexia and weight loss, but no night sweats.

On examination he appeared unwell and was in very severe pain, although with no focal spinal tenderness. All movements of the lumbar spine were reduced, with straight leg raising reduced on the left to $60^{\circ}$, with a positive sciatic stretch test. The left ankle jerk was absent. There were no abnormalities in the heart, lungs or abdomen.

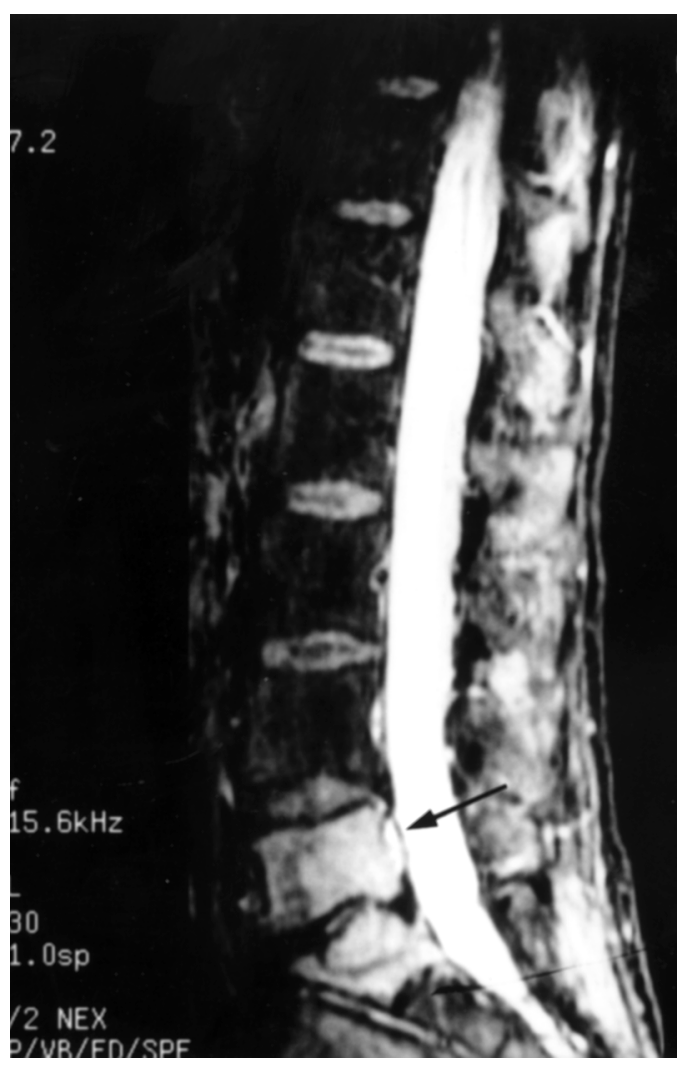

Figure 1 Patient 1. Magnetic resonance imaging of the lumbar spine with a sagittal STIR sequence showing abnormal pathological high signal in the bodies of L5 and S1.
Blood tests showed a high erythrocyte sedimentation rate (ESR) of $91 \mathrm{~mm} 1 \mathrm{st} h$ with an anaemia of chronic disease. $\mathrm{C}$ reactive protein (CRP) was high at $70 \mathrm{mg} / \mathrm{l}$, liver function tests, including alkaline phosphatase, and urea and electrolytes were normal, as were calcium and prostate specific antigen. Urine analysis on dipstick testing was normal. Although initial radiographs of the lumbar spine were unremarkable, apart from disc space narrowing only at level L5/S1, magnetic resonance imaging of the lumbar spine showed extensive abnormal signal within L5/S1 consistent with a malignancy (fig 1) and abnormal tissue surrounding the left $\mathrm{L} 5$ and $\mathrm{S} 1$ nerve roots.

Abdominal ultrasound scan showed a probable carcinoma of the left kidney with a $4 \mathrm{~cm}$ lesion noted. Computed tomography of the chest and abdomen (fig 2) confirmed the renal lesion and showed bone deposits at T5, the left first rib, L4/L5 and S1. Left kidney biopsy was attempted, but insufficient material was available for analysis. A bone biopsy confirmed a poorly differentiated renal carcinoma. The patient died two months later.

PATIENT 2

This 70 year old woman presented with a four month history of low back pain that radiated into both groins, down the posterior aspect of both thighs to involve the knees, and as far as the feet. Morning stiffness was considerable. She was anorexic without weight loss.

She had no previous history of low back pain.

On examination, evidence was found of nodal generalised osteoarthritis. The lumbar spine showed a mild lumbar scoliosis but with a very good range of movement. Neurological examination of the legs showed normal straight leg raising, without focal tenderness, but subjective decrease in sensation in the right leg with bilateral absent ankle jerks. The rest of the examination was insignificant. A tentative diagnosis of nodal generalised osteoarthritis with lumbar spondylosis producing bilateral sciatica was made. Magnetic resonance imaging of the thoracic and lumbar spine showed very marked disc prolapse and protrusion at the level of L3/L4, involving both L4 nerve root sheaths. There was also disc prolapse at L2/L3 and, to a lesser extent, at T8 /T9. 


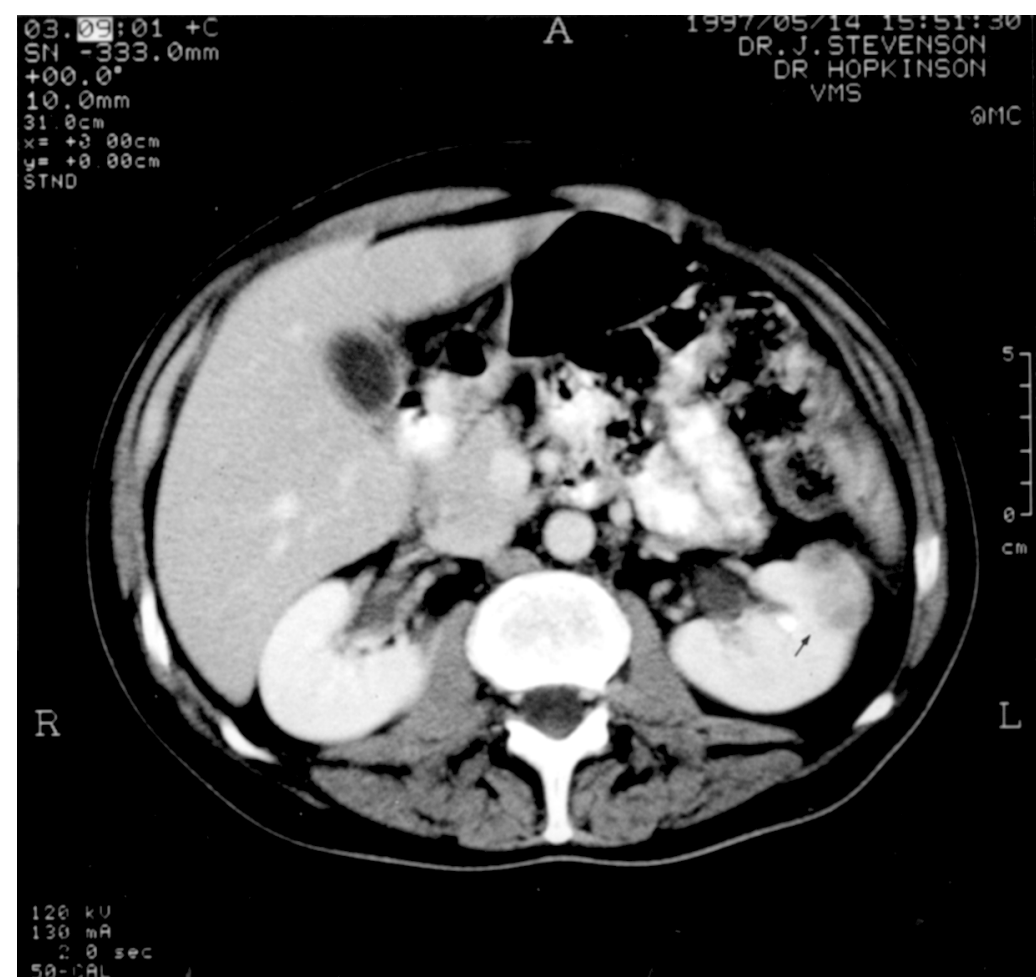

Figure 2 Patient 1. Post contrast computed tomography through the kidneys showing a mass in the lateral aspect of the left kidney.

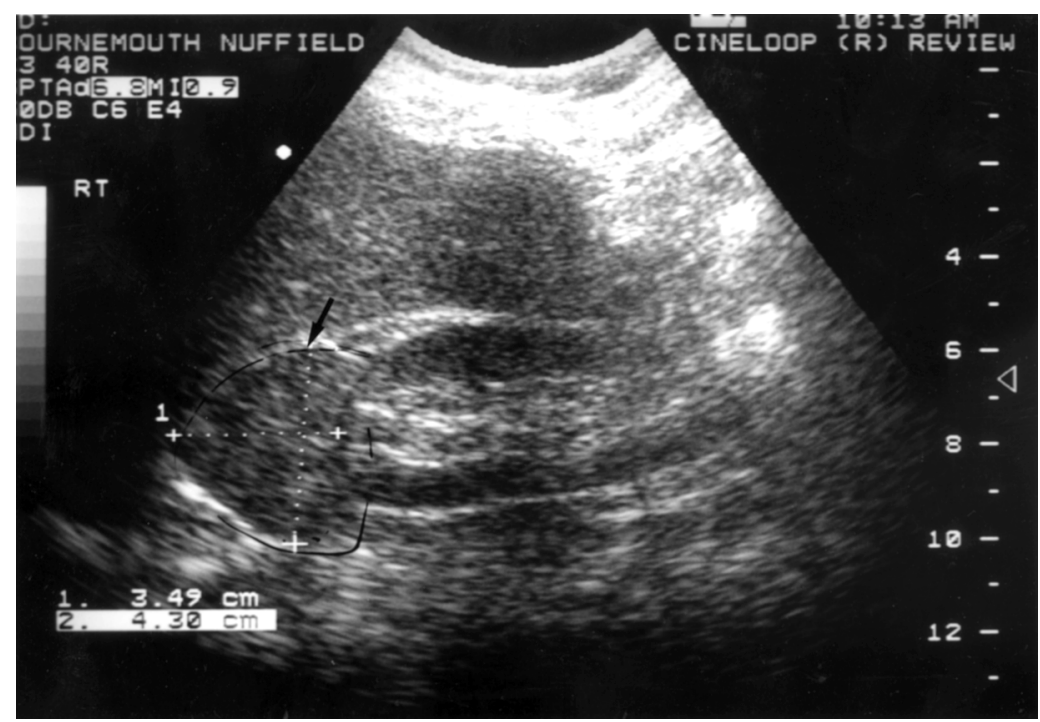

Figure 3 Patient 2. Ultrasound showing a 3.5 by $4.3 \mathrm{~cm}$ solid mass lesion arising from the upper pole of the right kidney.
However, because of the unusual history, abdominal ultrasound (fig 3) was performed, which showed a $4.5 \mathrm{~cm}$ renal mass, confirmed by computed tomography (fig 4 ).

A right nephrectomy was performed and histological examination showed a clear cell carcinoma of the renal cortex, still covered by the capsule.

Despite initial improvement in symptoms, muscular pain and stiffness returned to the pelvic and shoulder girdles, as the prednisolone dose was reduced over three months, with a corresponding increase in ESR. She was euthyroid at this time. Abdominal ultrasound showed no abnormality at the site of the nephrectomy and no lymphadenopathy, and the chest radiograph was clear. Symptoms settled well with an increase in corticosteroids.

\section{Discussion}

Renal tumours account for $1.6 \%$ of all malignancies. ${ }^{1}$ Renal cell carcinoma represents $90 \%$ of adult renal neoplasm ${ }^{2}$ and in less than $10 \%$ of these patients, their symptoms are those of metastatic disease. To our knowledge the "pick-up rate" of renal cell carcinoma by abdominal ultrasound has not been established, but will depend on tumour size and position, operator skill and body habitus.

We have described two patients presenting to the rheumatology clinic with a final diagnosis of renal cell carcinoma. The classic triad of renal cell carcinoma are haematuria, loin pain and a palpable mass, ${ }^{3}$ but neither of these patients had such "local" symptoms. A high index of suspicion is therefore needed to diagnose this disease.

In patients presenting with sciatica, secondary to bony destruction, pain is often extreme, night pain is prominent and there may be other suggestive symptoms such as anorexia, weight loss and night sweats. Investigations may show a raised ESR and altered bone biochemistry. Plain radiographs may be unremarkable, as in the case of patient 1 , but magnetic resonance imaging will be diagnostic. The testicular pain felt by patient 1 was probably referred pain directly from metastatic disease.

In patients with symptoms suggestive of polymyalgia rheumatica, underlying renal malignancy should always be considered. When there is a poor response to corticosteroids, either clinically and/or by ESR, further investigations are required. ${ }^{45}$ Abdominal ultrasound together with chest radiograph are then important.

Polymyalgic symptoms can resolve after successful removal of the underlying tumour. Often, however, metasteses are already evident. In the case of patient 2 the return of polymyalgic symptoms despite lack of secondary tumour, suggests that the two conditions may have been coincidental.

\section{The lesson}

- A high index of suspicion is needed to diagnose renal cell carcinoma, as it often does not present as the classic triad of haematuria, loin pain and a palpable mass.

- You should always be wary of new onset back pain in the elderly.
Prednisolone, $15 \mathrm{mg} /$ day, was started with subsequent improvement of ESR and CRP. 


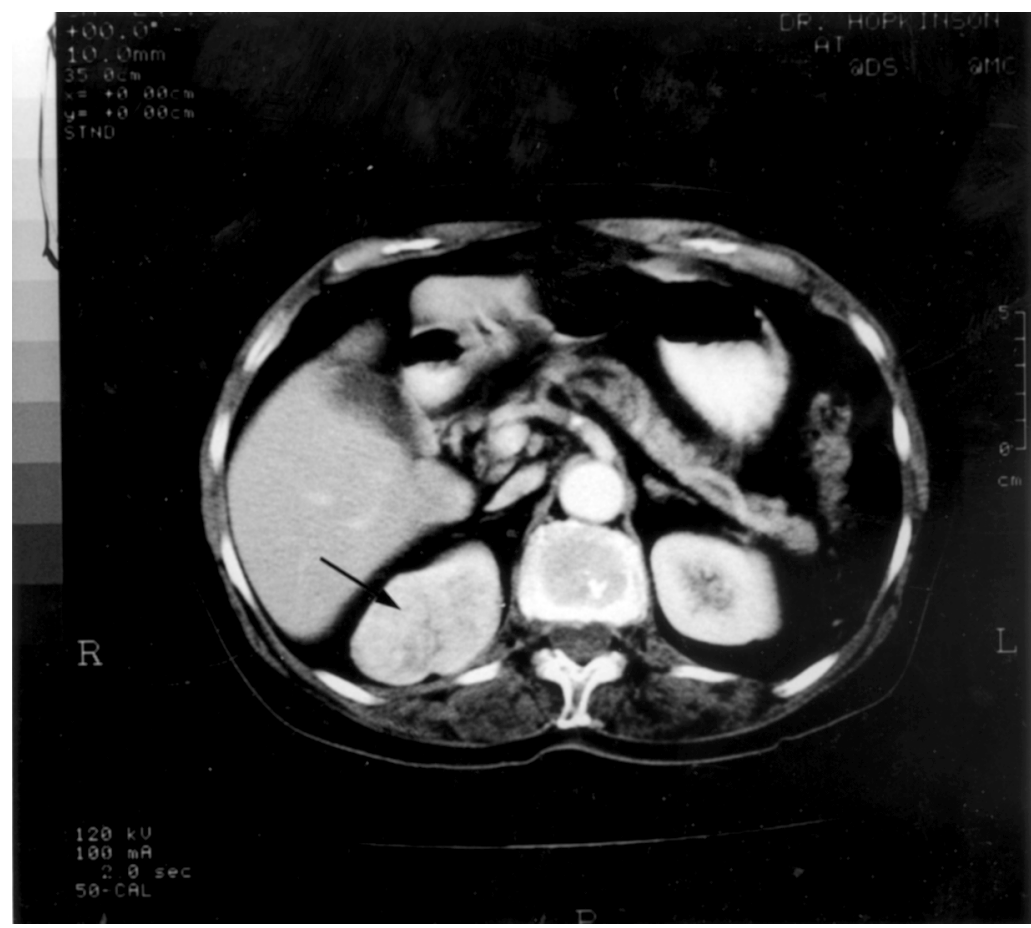

Figure 4 Patient 2. Computed tomography of abdomen, confirming the right sided renal tumour.
- In patients with polymyalgia rheumatica, if there is a poor response to corticosteroids, further investigations, such as abdominal ultrasound, should always be considered.

- Abdominal ultrasound is an important tool for establishing a diagnosis of renal cell carcinoma.

- Normal plain radiographs do not exclude bony metastatic disease.

We would like to thank Dr J Stevenson, Consultant Radiologist, Royal Bournemouth and Christchurch Hospitals, for his help with imaging.

1 Gore ME. Advances in management of renal cell carcinoma. In: Hendry W, Kirkby R, eds. Recent advances in urology/andrology. Edinburgh: Churchill Livingstone, 1993:81.

2 Chapman S., Nakielny R. Aids to radiological differential diagnosis 3rd ed. London: WB Saunders Company,1995: 308.

3 Ool GC, Sagar G, Lynch D, Arkell DG, Ryan PG. Cystic renal cell carcinoma: radiological features and clinico-pathological correlation. Clin Radiol 1996;51: 791-6.

4 Brooks RC, McGee SR. Diagnostic dilemmas in polymyalgia rheumatica. Arch Intern Med 1997;157:162-8.

5 Sidhom OA, Basalaev M, Sigal LH. Renal cell carcinoma presenting as polymyalgia rheumatica. Resolution after nephrectomy. Arch Intern Med1993;153:2043-5. 